\title{
Acute Groin Pain in an Adolescent Sprinter: A Case Report
}

\author{
Ann-Maria Byrne ${ }^{\mathrm{a}, \mathrm{b}}$, Declan Reidy
}

\begin{abstract}
Avulsion fractures of the lesser trochanter apophysis are rare injuries due to strenuous musculo-skeletal activity in sporting adolescents. The tendons and ligaments of the immature skeleton are able to withstand greater tensile forces than the vulnerable developing apophysis, thus resulting in these avulsion injuries associated with vigorous eccentric musculo-skeletal contractions. We report the case of a 13-year-old boy who presented with acute onset of limp and groin pain following sprinting. Radiographs revealed an acute avulsion of the lesser trochanter of the proximal femur. Full recovery followed a period of protected weight-bearing and symptomatic treatment.
\end{abstract}

Keywords: Adolescent; Avulsion; Apophysis; Lesser trochanter

\section{Introduction}

Avulsion fractures of the apophyses are rare injuries caused by sudden, vigorous or eccentric musculo-skeletal activity, while the subject is engaged in a strenuous sporting event

Manuscript accepted for publication January 19, 2012

${ }^{a}$ Department of Trauma and Orthopaedics, Our Lady of Lourdes Hospital, Drogheda, Co. Louth, Ireland

${ }^{\mathrm{b}}$ Corresponding author: 33 Addison Ave, Addison Park, Glasnevin, Dublin 11, Ireland. Email: ann maria byrne@hotmail.com

doi:10.4021/ijcp3w such as gymnastics, jumping or sprinting. The apophysis is a secondary centre of ossification contributing to the size and contour but not to the length of bone. Apophyses are the sites of origins or insertions of major muscle groups and in the immature skeleton, tendons and ligaments are able to withstand greater force than the developing bone. The variation in apophyseal ossification, and decreased elasticity of physeal cartilage results in the apophysis being susceptible to avulsion injuries in sporting adolescents. We report the case of a 13-year-old adolescent sprinter who presented following a competition related acute avulsion of the lesser trochanter of the proximal femur.

\section{Case Report}

A 13-year-old boy was referred to the fracture clinic with a two day history of right groin pain associated with a limp. Onset of these symptoms occurred acutely following a sprinting competition at school. He was unable to complete the race due to the acute onset of groin pain and limped to the sideline. As his symptoms did not improve the following day, his mother brought him to the emergency department where he underwent routine investigations of a painful limp for his age group. He was systemically well and no abnormalities were found on examination of his haematological and inflammatory markers. Anteroposterior pelvis radiography with 'frog-leg' lateral images was carried out to investigate for signs of possible Slipped Upper Femoral Epiphysis or Perthes' disease. He was discharged with analgesia and referred to the fracture clinic the following morning.

On entering the consultation room, he was noted to have a right-sided limp consistent with an antalgic gait. Abdominal examination was normal but he was found to have pain on passive flexion and internal rotation of his right hip, and was unable to actively flex at the hip joint. While was no external evidence of trauma, he had point tenderness in the region of his right lesser trochanter. Distal neurovascular examination was normal. Review of the radiographs revealed an acute avulsion of the lesser trochanteric apophysis (Fig. 1). The avulsed fragment of the lesser trochanter had been displaced cephalad by $1 \mathrm{~cm}$ due to the pull of the tendon of 


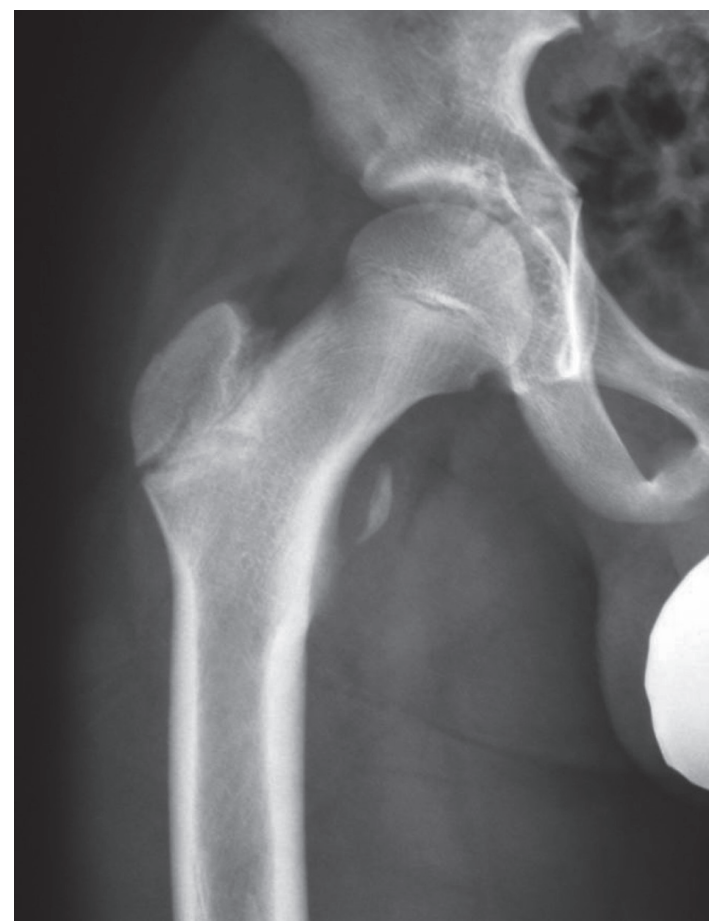

Figure 1. Apophyseal avulsion fracture of lesser trochanter with proximal displacement of fracture fragment (marked with arrow).

the iliopsoas muscle.

Treatment was symptomatic with analgesia and a period of non-weight bearing for two weeks with crutches. After this time, he commenced a further two week period of protected weight bearing building up to full weight bearing as tolerated under the guidance of our physiotherapy colleagues. At twelve-week follow-up, there was no radiographic evidence of further fragment displacement and his symptoms had completely resolved. He returned to full athletic activity with no recurrence of his limp or groin pain.

\section{Discussion}

Avulsion injuries in athletic adolescents are found most commonly about the pelvis and proximal femur. The most common reported site is avulsion of the ischial tuberosity, followed by the anterior superior iliac spine, anterior inferior iliac spine, lesser trochanter, and rarely, avulsion of the greater trochanter of the femur [1]. Although not as common as other avulsions in the pelvis, lesser trochanter avulsion causes considerable pain and decreased function. In adults, however, such avulsions are rare and are virtually always secondary to metastatic bone disease [2].The main cause of avulsion of the lesser trochanter in adolescents is forceful contraction of the iliopsoas tendon during hip flexion. The excessive stress concentration at the site of the iliopsoas muscle leads to a tensile failure of the apophysis of the lesser trochanter. These injuries are usually seen between the ages of seven and sixteen years, most commonly occurring at fourteen years of age. Due to fusion of the lesser trochanteric apophysis, the age-limit for these injuries occurs at eighteen or nineteen years $[1,3]$.

The diagnosis may be suggested by the patient's age and mechanism of injury. However, thorough workup for other causes of paediatric hip pathology must be carried out to avoid the devastating sequelae of undiagnosed septic arthritis, slipped capital femoral epiphysis and Perthes' disease in this age group. Groin pain and limp with little external evidence of trauma is the most frequent presenting scenario. Radiographic views of the proximal femur will demonstrate the avulsed fragment of the lesser trochanter and is classified as a Salter-Harris I fracture. The fragment is displaced proximally by the pull of the iliopsoas muscle. However, most apophyseal avulsion fractures of the lesser trochanter do not displace significantly as the periosteum is usually in continuity with the fragment [4]. A femoral metaphysic bone flake may accompany the avulsed lesser trochanter, particularly in patients reaching skeletal maturity when the growth plate becomes less active [1].

It has been suggested that surgical intervention should be considered with fragment displacement of greater than 2 $\mathrm{cm}$, if the fragment is of sufficient size to hold hardware. Quarrier and Wightman [5] reported the case of a ballet dancer who experienced 6 years of chronic hip pain and dysfunction. Review of the initial pelvic radiographs demonstrated an avulsion fraction of the lesser trochanter which had not been treated. Ultimately, surgical excision of the ossicle eliminated the hip pain, and their patient returned to full activity. However, Fernback and Wilkinson have shown that the amount of displacement does not correlate with presenting symptoms nor with time to recovery. Theologis et al. [6] reported early and complete recovery following symptomatic treatment even in patients with marked proximal displacement of the avulsed segment of the lesser trochanter. Our recommendation is symptomatic treatment with protected weight-bearing on crutches for 3 to 4 weeks and analgesia as required. Early return to sports has been associated with risk of recurrent injury and chronic groin pain. Hence, sporting activities should be avoided until healing has completed which could take up to 12 weeks. Surgical intervention for avulsion fractures is only indicated where a non-union or fibrous union has occurred with motion at the fracture site resulting in chronic pain $[1,7]$. However, the majority of patients become asymptomatic and return to original activity levels even with persistent radiological abnormalities [1].

\section{Competing Interest}

No conflicts of interest exist for the author. 


\section{References}

1. Fernbach SK, Wilkinson RH. Avulsion injuries of the pelvis and proximal femur. AJR Am J Roentgenol. 1981;137(3):581-584.

2. Afra R, Boardman DL, Kabo JM, Eckardt JJ. Avulsion fracture of the lesser trochanter as a result of a preliminary malignant tumor of bone. A report of four cases. J Bone Joint Surg Am. 1999;81(9):1299-1304.

3. Tachdjian, M. O. (1990) Pediatric Orthopedics, 2nd ed. W. B. Saunders, Philadelphia, pp 3247.

4. Anderson K, Strickland SM, Warren R. Hip and groin injuries in athletes. Am J Sports Med. 2001;29(4):521533.

5. Quarrier NF, Wightman AB. A ballet dancer with chronic hip pain due to a lesser trochanter bony avulsion: the challenge of a differential diagnosis. J Orthop Sports Phys Ther. 1998;28(3):168-173.

6. Theologis TN, Epps H, Latz K, Cole WG. Isolated fractures of the lesser trochanter in children. Injury. 1997;28(5-6):363-364.

7. Schlonsky J, Olix ML. Functional disability following avulsion fracture of the ischial epiphysis. Report of two cases. J Bone Joint Surg Am. 1972;54(3):641-644. 